\title{
In Memoriam: Recordando a Ernesto Pollitt Burga (1938-2016)
}

\author{
In Memoriam: Remembering Ernesto Pollitt Burga \\ (1938-2016)
}

\author{
Tomás P. Caycho \\ Universidad de San Martín de Porres, Lima, Perú.
}

Doctorando en Psicología. Docente de Métodos de Investigación y Psicología experimental e Investigador Interno del Instituto de Investigación.

Recibido: 12-01-16

Aprobado: 01-02-16

\section{Correspondencia}

Email: tcaycho@usmp.edu.pe

\section{Citar como:}

Caycho, T. (2016). In Memoriam: Recordando a Ernesto Pollitt Burga (1938-2016). Propósitos y Representaciones, 4(1), 385-396. doi: http://dx.doi.org/10.20511/pyr2016. v4n1.94

(c) Universidad San Ignacio de Loyola, Vicerrectorado de Investigación y Desarrollo, 2016. (c) BY-NC-ND Este artículo se distribuye bajo licencia CC BY-NC-ND 4.0 Internacional (http://creativecommons.org/licenses/by-nc-nd/4.0/). 


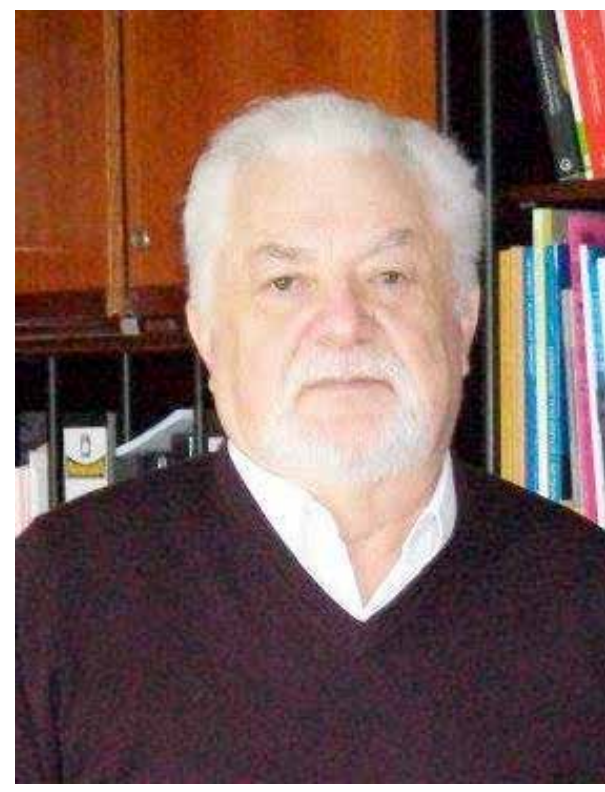

El año 2016 se inicia con una lamentable noticia para la comunidad psicológica nacional e internacional. En la madrugada del 5 de enero falleció el doctor Ernesto Pollitt Burga, psicólogo peruano con más de 50 años de investigaciones ininterrumpidas acerca del desarrollo infantil. Sus investigaciones -publicadas en las más prestigiosas revistas de psicología, pediatría y nutrición-, sus libros, sus cualidades humanas y sus aportes teóricos y metodológicos hablan de la trascendencia de su obra y justifican estas breves líneas acerca de su vida y obra.

Ernesto Pollitt nació el 21 de abril de 1938 en Lima. Ingresó a la Facultad de Letras de la Pontificia Universidad Católica del Perú en 1956. Inicialmente cursó estudios de Derecho, para luego pasar a la carrera de Filosofía y, finalmente, seguir estudios de Psicología, donde tuvo como docentes a importantes figuras de la psicología y la psiquiatría peruana de la época, como Baltazar Caravedo, Arnaldo Cano y Emilio Majluf. Viajó a los Estados Unidos para incorporarse como investigador asistente del Dr. Jhon Money en la Unidad de Psicoendocrinología de la Universidad John Hopkins. Con Money publicaría trabajos referidos a la psicología del enanismo (Pollitt \& Money, 1964; Money \& Pollitt, 1966) y a la ambigüedad citogenética y psicosexual (Money \& Pollitt, 1964).

Luego de dos años de trabajo con Money, Pollitt retorna al Perú para viajar a la ciudad de Chiclayo con el objetivo de investigar el impacto de la 
administración de alimentos fortificados con proteínas sobre el desarrollo de niños desnutridos. Este hecho marcó en Pollitt la decisión de dedicarse a investigar las consecuencias de la desnutrición en el desarrollo infantil. Cursó estudios de Psicología Evolutiva en la Universidad de Cornell (Nueva York), trabajando tiempo después en el Departamento de Pediatría de la Universidad de Yale y en el Child Study Center. Años más tarde, Pollitt ingresó a laborar en el Instituto de Investigaciones de las Naciones Unidas para el Desarrollo Social (UNRISD), en Ginebra. Es por estos años que aparecen sus primeros trabajos acerca del desarrollo motor y mental en niños con malnutrición calórica-proteica severa (Pollitt \& Granoff, 1967) y respecto al papel de variables biológicas y sociales en relación con la estatura en niños de barriadas de Lima (Pollitt \& Ricciuti, 1969). Temas que serían abordados posteriormente en otras publicaciones (Pollitt, Gilmore \& Valcarcel, 1978; Pollitt \& Wirtz, 1981; Pollitt, 2008a).

Ejerció la docencia como Profesor Asociado del Departamento de Nutrición y Ciencias de la Alimentación del Instituto Tecnológico de Massachusetts (MIT), y como Profesor Asociado y Titular en la Universidad de California en Davis, de la cual es Profesor Emérito. Durante estos años realizó investigaciones en Egipto, Guatemala, India e Indonesia acerca de los efectos de alimentos fortificados, así como sobre los efectos a largo plazo de la desnutrición en el desarrollo, desde la gestación hasta la adultez (Gorman \& Pollitt, 1993; Pollitt, Saco, Laibel \& Viteri, 1986; Pollitt, Gorman, Engle, Martorell \& Rivera, 1993). Otros temas de interés de Pollitt en los últimos años fueron la influencia de los estados pluricarenciales de niños sobre la capacidad de toma de decisiones (Pollitt, 2008b) y la fundamentación de los hitos del desarrollo motor grueso como indicadores del desarrollo general de los niños menores de 2 años (Pollitt \& Bacallao, 2009; Pollitt \& Caycho, 2010; Pollitt, 2012).

Debido a sus importantes contribuciones, el Dr. Pollitt ha sido consultor de diversas instituciones peruanas y extranjeras. Además, por 
sus aportes, ha recibido diversos reconocimientos: Profesor Visitante Sir Clavering Fisson en la Universidad de Londres; Premio Internacional de la Salud Pública Príncipe Mahidol, en el año 2000, otorgado por la Fundación Mahidol de la Casa Real de Tailandia, en Bankgok; Premio en Nutrición de la Academia Americana de Pediatría (1999); Premio de Excelencia en Investigación de la Sociedad Americana en Nutrición Internacional (1998); Profesor Honorario de la Pontificia Universidad Católica del Perú (1998); Miembro Emérito de la Sociedad Latinoamericana de Nutrición (2003); Premio Nacional de Nutrición de la Sociedad Peruana de Nutrición (2007); Profesor Honorario de la Universidad Ricardo Palma (2007); Miembro de la Academia Nacional de Ciencias (2009), siendo el único psicólogo peruano dentro de esta prestigiosa organización académica, entre otros.

Entre las principales contribuciones del trabajo del Dr. Pollitt se puede mencionar, en primer lugar, sus estudios sobre los efectos de las deficiencias calórico-proteicas y de hierro en el desarrollo infantil, demostrando las consecuencias negativas de la anemia ferropénica en el aprendizaje y el desarrollo motor; resultados que han generado, a lo largo de diversos países, iniciativas de implementación de políticas públicas (Cueto, 2005). En segundo lugar, demostró que los efectos de la desnutrición pueden revertirse de acuerdo con el tipo de déficit nutricional, su duración y la edad de padecimiento. En tercer lugar, Pollitt concibe el desarrollo como un proceso probabilístico en el cual intervienen factores internos y externos al organismo en constante interacción. Por último consideró, de entre las diversas dimensiones del desarrollo psicobiológico del niño, a los hitos motores que emergen durante los primeros 24 meses de vida como los mejores predictores del desarrollo intelectual y el rendimiento académico.

La vida del Dr. Pollitt no podría entenderse sin la presencia de dos pilares fundamentales: la Dra. Carmen Saco-Pollitt, su esposa desde 1970, y Ricardo Pollitt Saco, su primer y único hijo, doctor y especialista en 
epidemiología del Hospital de la Universidad de Stanford. Ambos han sido sus principales motivaciones.

Para terminar con un tono personal, quien escribe estas líneas conoció al Dr. Pollitt en el verano del 2009, siendo seleccionado por él, durante casi dos años, como su asistente de investigación; tiempo durante el cual pude conocer su sencillez, humildad, laboriosidad y disciplina para con el trabajo. Él me enseñó que ser psicólogo y científico es posible y que solo la investigación científica ayudará al desarrollo de nuestros pueblos. Aún recuerdo sus palabras de aliento y sus innumerables anécdotas, que buscaban no solo contribuir con el trabajo académico en marcha, sino también con mi desarrollo personal. Fui afortunado de poder estar cerca del psicólogo, investigador y hombre que, con su legado y consejos, seguirá inspirando a muchas generaciones.

\section{Referencias}

Cueto, S. (2005). Ernesto Pollitt: El hombre de hierro. En Asociación de Egresados y Graduados (Ed.). VIII Ceremonia de Homenaje a Ex Alumnos Distinguidos (pp. 29-31). Lima: Asociación de Egresados y Graduados Distinguidos de la PUCP.

Gorman, K. S., \& Pollitt, E. (1993). Determinants of school performance in Guatemala: Family background characteristics and early abilities. International Journal of Behavioral Development, 16, 75-91.

Money, J., \& Pollitt, E. (1964). Cytogenetic and psychosexual ambiguity. Archives of General Psychiatry, 11, 589-595.

Money, J., \& Pollitt, E. (1966). Studies in the psychology of dwarfism II. Personality maduration and response to growth hormone treatment in hypopituitary dwarfs. Journal of Pediatrics, 65, 381-390.

Pollitt, E. (2008a). La historia de la madre pero no sus años de escolaridad predicen el rendimiento escolar de las hijas en una población crónicamente pobre. Teoría e Investigación en Psicología, 17(1), 107-118. 
Pollitt, E. (2008b). La pobreza y la libertad en la toma de decisiones durante la infancia. En Ruiz, P., Patrón, P. y Quintanilla, P. (Eds.). Desarrollo humano y libertades. Una aproximación interdisciplinaria (pp. 159178). Lima: Pontificia Universidad Católica del Perú.

Pollitt, E. (2012). Estabilidad y variabilidad en la adquisición de seis hitos motores durante la infancia temprana. Revista de Psicología de la PUCP, $30(2), 407-429$.

Pollitt, E., \& Bacallao, J. (2009). La utilización de hitos motores en estudios poblacionales como indicadores del desarrollo en niños menores de dos años. Lima: Organización Panamericana de la Salud.

Pollitt, E., \& Caycho, T. (2010). Desarrollo motor como indicador del desarrollo infantil durante los dos primeros años de vida. Revista de Psicología de la PUCP, 28(2), 385-413.

Pollitt, E., Gilmore, M., \& Valcárcel, M. (1978). Early mother infant interaction and somatic growth. Early human development, 1, 325-330.

Pollitt, E., Gorman, K. S., Engle, P. L., Martorell, R., \& Rivera, J. (1993). Early supplementary feeding and cognition. Monograph of the Society for Research in Child Development, 58(7), 1-99.

Pollitt, E., \& Granoff, D. (1967). Mental and motor development of Peruvian children treated for severe milnutrition. Interamerican Journal of Psychology, 1(2), 92-103.

Pollitt, E., \& Money, J. (1964). Studies in the psychology of dwarfism. Intelligence quotient and school achievement. Journal of Pediatrics, 64, 415-421.

Pollitt, E., \& Ricciuti, H. (1969). Biological and social correlates of stature among children in the slums of Lima, Perú. American Journal of Orthopsychiatry, 39(5), 735-747.

Pollitt, E., Saco, C., Leibel, R. L., \& Viteri, F. E. (1986). Iron defiency and behavioral development in infants and pree-school children. American Journal of Clinical Nutrition, 43, 555-565.

Pollitt, E., \& Wirtz, S. (1981). Mother-infant feeding interaction and weight gain in the first month of life. Journal of the American Dietary Association, 78, 596-601. 